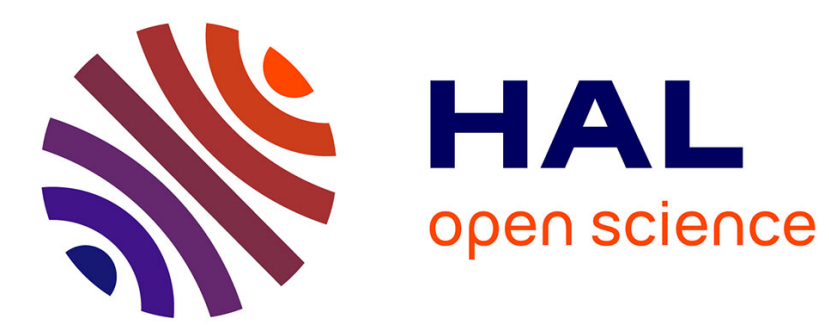

\title{
New signal processing technique for density profile reconstruction using reflectometry
}

F. Clairet, B. Ricaud, F. Briolle, S. Heuraux, C. Bottereau

\section{To cite this version:}

F. Clairet, B. Ricaud, F. Briolle, S. Heuraux, C. Bottereau. New signal processing technique for density profile reconstruction using reflectometry. Review of Scientific Instruments, 2011, 82, pp.3502. 10.1063/1.3622747 . hal-00961734

\section{HAL Id: hal-00961734 https://hal.science/hal-00961734}

Submitted on 21 Mar 2014

HAL is a multi-disciplinary open access archive for the deposit and dissemination of scientific research documents, whether they are published or not. The documents may come from teaching and research institutions in France or abroad, or from public or private research centers.
L'archive ouverte pluridisciplinaire HAL, est destinée au dépôt et à la diffusion de documents scientifiques de niveau recherche, publiés ou non, émanant des établissements d'enseignement et de recherche français ou étrangers, des laboratoires publics ou privés. 


\title{
New signal processing technique for density profile reconstruction using reflectometry
}

\author{
F. Clairet, ${ }^{1}$ B. Ricaud,${ }^{1,2}$ F. Briolle, ${ }^{2,3}$ S. Heuraux,${ }^{4}$ and C. Bottereau ${ }^{1}$ \\ ${ }^{1}$ CEA, IRFM, F-13108 Saint-Paul-lez-Durance, France \\ ${ }^{2}$ CPT UMR 6207, Campus de Luminy, case 907, F-13288 Marseille, France \\ ${ }^{3}$ CReA, BA 701, F-13306 Salon de Provence, France \\ ${ }^{4} I J L-P 2 M$, UMR-CNRS 7198, Université Henri Poincaré, F-54506 Vandoeuvre, France
}

(Received 11 March 2011; accepted 14 July 2011; published online 25 August 2011)

\begin{abstract}
Reflectometry profile measurement requires an accurate determination of the plasma reflected signal. Along with a good resolution and a high signal to noise ratio of the phase measurement, adequate data analysis is required. A new data processing based on time-frequency tomographic representation is used. It provides a clearer separation between multiple components and improves isolation of the relevant signals. In this paper, this data processing technique is applied to two sets of signals coming from two different reflectometer devices used on the Tore Supra tokamak. For the standard density profile reflectometry, it improves the initialization process and its reliability, providing a more accurate profile determination in the far scrape-off layer with density measurements as low as $10^{16} \mathrm{~m}^{-1}$. For a second reflectometer, which provides measurements in front of a lower hybrid launcher, this method improves the separation of the relevant plasma signal from multi-reflection processes due to the proximity of the plasma. (C) 2011 American Institute of Physics. [doi:10.1063/1.3622747]
\end{abstract}

\section{INTRODUCTION}

Reflectometry relies on the fact that as an electromagnetic wave propagates through the plasma, its phase is shifted due to the deviation of the local refractive index from the vacuum value. At a certain critical density corresponding to the cut-off layer, in a WKB description (or under WKB assumptions), this refractive index goes to zero and the probing wave is reflected. The In and Quadrature phase detection of the reflected wave provides a complex signal from which amplitude and phase variation can be measured separately. The density profiles are then reconstructed from the detected phase according to a recursive numerical algorithm. ${ }^{1}$ Among the traditional filtering techniques and Fourier analysis to recover the relevant plasma echo, many signal analysis procedures have been extensively tested most of the time to compensate for the lack of signal and phase scrambling due to the turbulence. ${ }^{2-4}$ This latter problem has been overcome over the past decade by using fast sweep heterodyne techniques, ${ }^{5}$ which provide reliable and accurate phase measurements. However, reflectometry signals exhibit a multi-frequency nature and the extraction of the relevant plasma echo can, in some circumstances, appear to be delicate. As a matter of fact, the signal analysis can become problematic when multi-reflection processes occur simultaneously. Recently, a new tool ${ }^{6}$ based on a tomographic technique in the time-frequency plane has been developed and it substantially improves the traditional Fourier analysis in separating echoes with a similar frequency but over different time domains with a great precision. While Fourier transform is based on the projection onto a basis of sine and cosine functions, the tomogram transform consists in a decomposition of the signal onto an orthonormal basis of linear chirps. In this paper we illustrate the performance of the tomographic analysis applied to Tore Supra (TS) reflectometry signals through various examples. We first present, in Sec. II, the theoretical aspects of the tomogram technique to motivate its application to improve the signal extraction corresponding to the first plasma reflection and to provide a better rejection of multi-reflections. In Secs. III and IV, these two performances of the tomographic analysis are illustrated with two different reflectometer configurations. In Sec. III, we apply this analysis technique, to the standard density profile reflectometer, for the first X-mode cut-off detection. The problem that we encounter on TS is to separate the plasma scrapeoff layer echo from the back wall component. This problem can prevent a proper density profile initialization and modify substantially the reconstruction at the far edge. The second example, treated in Sec. IV, corresponds to data obtained using the same reflectometer but measuring density profiles in front of a lower hybrid current drive (LHCD) launcher. Two waveguides bring the wave directly inside the vacuum chamber at a few millimeters from the detected plasma edge. The multicomponent nature of the signal is particularly significant as the reflectometer antennas are very close to the plasma, which causes secondary multi-reflections. This reflectometer was installed for a short period of time for test purposes. Such a reflectometry configuration will occur on ITER to address at least two specific physic studies: the plasma positioning control with a plasma position reflectometry system, ${ }^{7,8}$ and, the power coupling to the plasma of additional powers such as ion cyclotron resonance heating (ICRH) or LHCD systems where measurements are foreseen in front of the launchers. ${ }^{9}$ Then, in Sec. V we discuss the advantages and limitations of this new signal processing technique, and directions for further research.

\section{TOMOGRAM}

\section{A. Definition}

The so-called tomogram transform was first defined in the context of quantum mechanics and operator theory. ${ }^{10,11}$ 

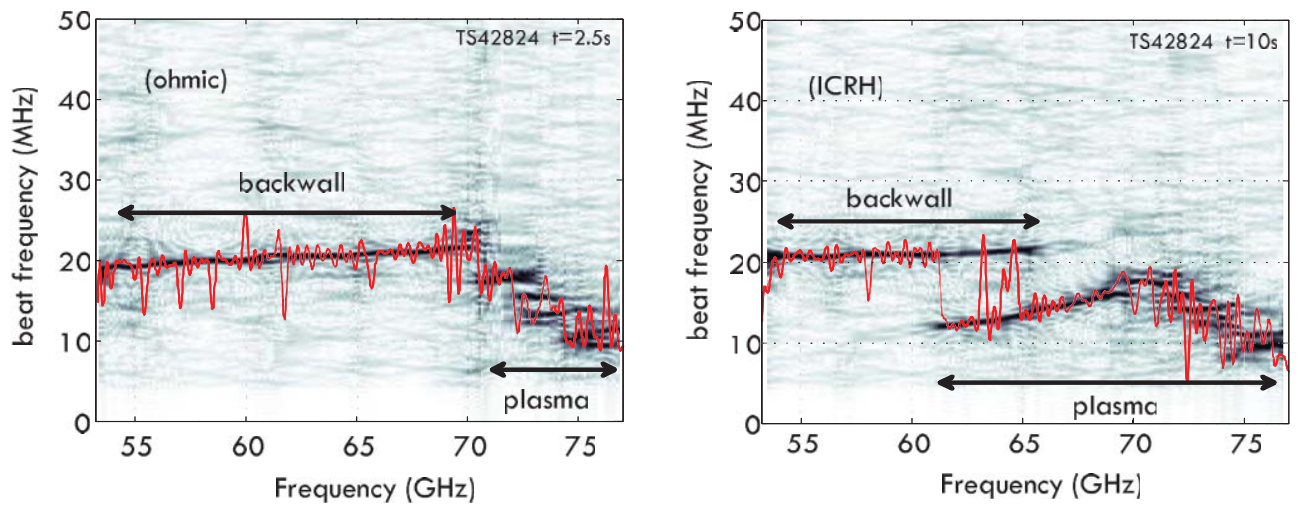

FIG. 1. (Color online) Short time window FFT analysis and instantaneous beat frequency (solid line) vs. probing frequency of a V-band reflectometer signal. Both signals have been recorded during the same plasma at different time without (left) and with (right) additional ICRH.

A self-adjoint operator depending on a parameter $\theta$ is introduced together with its eigenfunctions: the tomogram transform performs the projection over the basis formed by these eigenvectors. The properties of the operator ensure that the eigenfunctions are $\theta$-dependent and orthogonal for all fixed $\theta$. As a consequence, the signal can be projected, separated in parts, and each part can be re-synthesized in time in a fast and efficient way. Moreover, $\theta$ allows tuning the decomposition in order to be adapted to the signal. It allows a better separation of the different parts.

In the framework of Tore Supra reflectometry, the tomogram associated to the operator $\Xi(\theta)$ of Ref. 12 is used. It is the association of two parts, one related to time, the other related to frequency and a parameter $\theta \in[-\pi / 2, \pi / 2]$. The parameter $\theta$ allows to pass from the time representation of the signal $(\theta=0)$ to the frequency one $(\theta=\pi / 2)$ via intermediate representations. The operator $\Xi(\theta)$ is

$$
\Xi(\theta)=\cos \theta t+i \sin \theta \frac{d}{d t},
$$

where $t$ and $d / d t$ are, respectively, the $t$-multiplication operator and the derivative operator. Its eigenfunctions $\psi_{X}^{\theta}$, i.e., defined by $\Xi(\theta) \psi_{X}^{\theta}=X \psi_{X}^{\theta}$, where $X$ corresponds to an eigenvalue of the operator $\Xi(\theta)$, form an orthogonal basis. The reflectometry signals are of finite time-length $T$ and the $\psi_{X}^{\theta}$ on the domain $[0, T]$ have the following expressions:

$$
\psi_{X}^{\theta}(t)=\frac{1}{\sqrt{T}} \exp \left(-i\left(\frac{1}{2 \tan \theta} t^{2}-\frac{X}{\sin \theta} t\right)\right) .
$$

The associated eigenvalue takes discrete values,

$$
X=\frac{2 \pi \sin \theta}{T} n,
$$

where $n$ is an integer. The tomogram transform $M$ of a signal $u$ is defined by

$$
M_{u}(X, \theta)=\left|C^{\theta}(X)\right|^{2},
$$

where $C^{\theta}(X)$ is the scalar product:

$$
C^{\theta}(X)=\frac{1}{\sqrt{T}} \int_{0}^{T} u(t) \overline{\psi_{X}^{\theta}(t)} d t .
$$

Remark that this transform represents an energy density and for $\theta=0$ the tomogram is just $|u(t)|^{2}$, whereas for $\theta=\pi / 2$, it is the density of the Fourier transform $|U(\omega)|^{2}$. The general theory of self-adjoint operators gives the resynthesis of the signal via the following relation:

$$
u(t)=\sum_{X} C^{\theta}(X) \psi_{X}^{\theta}(t) .
$$

In fact, each $\psi_{X}^{\theta}$ is a linear chirp with phase derivative

$$
\phi^{\prime}(t)=-\frac{1}{\tan \theta} t+\frac{X}{\sin \theta}=2 \pi\left(s t+f_{0}\right) .
$$

If plotted in the time-frequency domain, $\psi_{X}^{\theta}$ is a ridge of slope $s=(-2 \pi \tan \theta)^{-1}$ and initial frequency (at $t=0$ ) $f_{0}=X / 2 \pi \sin \theta$.

Thus, the tomogram transform can be seen as the projection on a basis of linear chirps. In consequence, this transform should be appropriate in the case of reflectometry data where chirp-like components are embedded in a complex signal, as one can see, for instance, on the spectrogram of Fig. 1.

\section{B. Practical processing steps and implementation}

To extract the plasma reflection from the raw signal, the tomogram analysis is done in several steps.

\section{Choice of $\theta$}

The selection of $\theta$ is not straightforward and depends on the signal. As explained in Sec. II A, each $\psi_{X}^{\theta}$ is a linear chirp with phase derivative: $\phi^{\prime}(t)=s t+f_{0}$. Let us assume that the signal to extract is a linear chirp with slope $a$ and initial frequency $b$ : if $s$ is taken equal to $a$, the tomogram decomposition will give the maximum of its efficiency since all the chirp energy will be concentrated on one peak situated at $f_{0}$ $=b$. This would correspond to the orthogonal projection on $\psi_{X}^{\theta}$ with $\theta=\arctan (-1 / 2 a \pi)$ and $X=2 \pi \sin \theta$. The choice of the best $\theta$ is usually made a posteriori with the help of the tomogram plot: several projections are computed, associated to different arbitrary chosen $\theta$. Then a comparison of the results is done by plotting the values of the function of two variables $M_{u}$. In the present study, for a more intuitive approach, we have adopted the chirp point of view and we plot $M_{u}$ with respect to $\theta$ and $f_{0}$; an example is shown in Fig. 2. The 2D plot highlights the different parts embedded in the signal which can be extracted by the tomogram decomposition. The $\theta$ for 

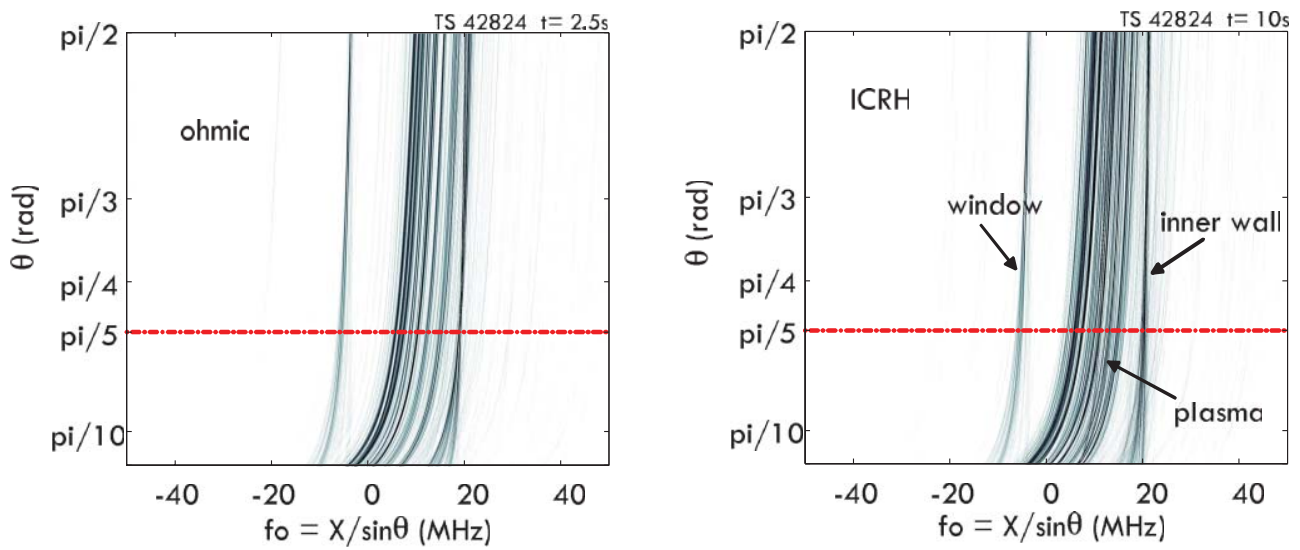

FIG. 2. (Color online) Tomograms of the ohmic (left) and ICRH (right) signals. The dashed line represents the parameter chosen for the projection at $\theta$ $=\pi / 5$.

which the separation between the different parts is the greatest is chosen for the extraction (see Sec. III). It is important to notice that the choice of the angle is robust, meaning that a small change of $\theta$ will lead to a small change of the energy pattern. This can be seen on the tomograms plotted in Fig. 2. For identical reasons, the same robustness holds if the signal to extract is not a perfectly linear chirp but possesses a small bending.

\section{Computation of the signal projections}

$C^{\theta}(X)$ : The reflectometry signal is discrete and contains $N=2000$ points per run of length $T=20 \mu \mathrm{s}$. The sampling frequency is $F_{s}=N / T=100 \mathrm{MHz}$. The relation (4) is here the discrete sum: $C^{\theta}(X)$ $=\sum_{n=1}^{N} u\left(\frac{n}{F_{s}}\right) \frac{1}{\sqrt{T}} \exp \left(i \frac{1}{2 \tan \theta} \frac{n^{2}}{F_{s}^{2}}\right) \exp \left(-i \frac{X}{\sin \theta} \frac{n}{F_{s}}\right)$. This quantity can be calculated using a fast Fourier transform (FFT) algorithm (when $N$ is a power of 2), as one can remark that the above equation is the discrete Fourier transform of the function $s\left(\frac{n}{F_{s}}\right) \exp \left(i \frac{1}{2 \tan \theta} \frac{n^{2}}{F_{s}^{2}}\right) \frac{1}{\sqrt{T}}$.

\section{Choice of sets of $C^{\theta}(X)$ corresponding to the plasma reflection}

For a given $\theta$, the energy density is calculated and plotted in Fig. 3. Several peaks of energy are present, which are the consequence of the multi-reflections contained in the signal. Using a threshold, regions with a high energy density are isolated. The set of frequencies $f_{0}$ corresponding to the plasma reflections is extracted. This gives the set $S$ of elements $X=f_{0} 2 \pi \sin \theta$, which will be used for the signal reconstruction.

\section{Extracted signals}

The reconstruction of the extracted signals is then performed according to the relation (5). For the plasma reflections, the sum is hence over the values of $X$ belonging to $S$.

The action of step 3 is to clear out the projection from the multi-reflection components of the signal. Then, the output of part 4 gives a temporal signal containing only the plasma reflections. The phase of this time-domain signal can then be used as the input of an algorithm for reconstructing the density profile.

\section{STANDARD DENSITY PROFILE REFLECTOMETRY}

A complete set of reflectometers, covering the frequency range from 33 to $150 \mathrm{GHz}$, provides density profile measurements on TS plasma from the edge to the centre and beyond. ${ }^{13,14}$ For normal operation, the reflectometer antennas are located in a dedicated porthole outside the

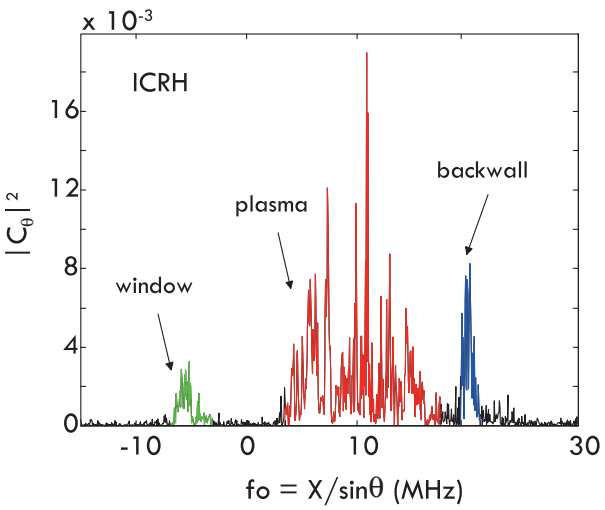

FIG. 3. (Color online) Slices of the tomogram at $\theta=\pi / 5$ for the ohmic signal (left) and the ICRH signal (right). Three components are present in the signal and correspond, respectively, to the quartz window, the plasma, and the back wall echoes. 

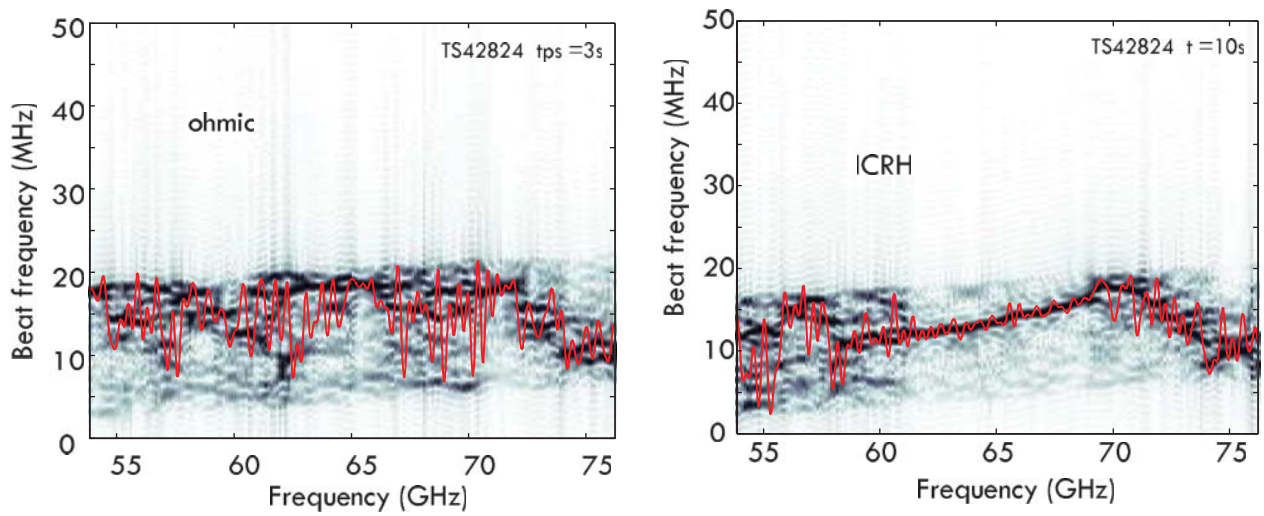

FIG. 4. (Color online) Spectrograms of the plasma signals separated by the tomographic analysis for ohmic and ICRH conditions (the solid lines correspond to a calculation from the instantaneous phase derivative).

vacuum vessel behind a quartz window. X-mode polarisation can provide an accurate initialization of the density profile as long as the magnetic field profile is known. The detection of the first cut-off frequency, which equals the electron cyclotron frequency at zero density, provides the starting position of the radial density profile. According the TS specificities (plasma size, magnetic field aspect ratio, etc.) during routine operation, the profile initialization is mainly performed with the V-band reflectometer signal, which is treated here. The reflectometer signal frequency, also called the beat frequency $\left(F_{b}\right)$, is related to the time derivative of the phase, such that

$$
F_{b}=\frac{1}{2 \pi} \frac{\partial \phi}{\partial t}
$$

The beat frequency can be determined either at a given point using the phase derivative at this point (the instantaneous beat frequency used to calculate the density profiles) or from sliding FFT which, in this case, realises an average over the time window analysis. Despite this averaging, the advantage of the FFT analysis is to conveniently provide a full picture of every signal components. In the following, several spectrograms are shown and are all constructed from the 2000 points signal by using short-term Fourier transform with windows of 100 points and a step of 10 points. Zero padding has been done using 1900 points to increase the smoothness of Fig. 1. Moreover, to make the different components visible even when the signal amplitude is low, the signal energy has been normalized to one for each window.

The scrape-off layer (SOL) region is characterized by outboard plasma with very low density $\left(\sim 10^{17} \mathrm{~m}^{-3}\right)$. Due to the low reflection efficiency of this very low edge density plasma, part of the probing wave crosses the cut-off layer and reflects onto the back wall (Fig. 1). Unfortunately, the frequency discrimination of these two echoes with traditional filtering techniques appears to be somewhat difficult due to their closeness in frequency. Figure 1 shows two different plasma edge behaviours according the experimental scenarios: under ohmic condition and with additional heating during the same plasma discharge.

Evidences of different plasma boundary conditions between the two plasma phases are observed. During the ohmic phase, the first plasma cut-off frequency occurs around 70 $\mathrm{GHz}$, while during the additional heating phase the cut-off appears above $60 \mathrm{GHz}$. In this latter case, additional plasma is created in the periphery probably due to some extra power deposition at the edge and/or because of the enhanced particle transport. When this edge plasma starts reflecting the wave, it does not screen totally the back wall because of its low density. The beat frequency increases continuously from 10 to $20 \mathrm{MHz}$ with the probing wave frequency. A change in the reflection behaviour appears above $68 \mathrm{GHz}$ where the beat
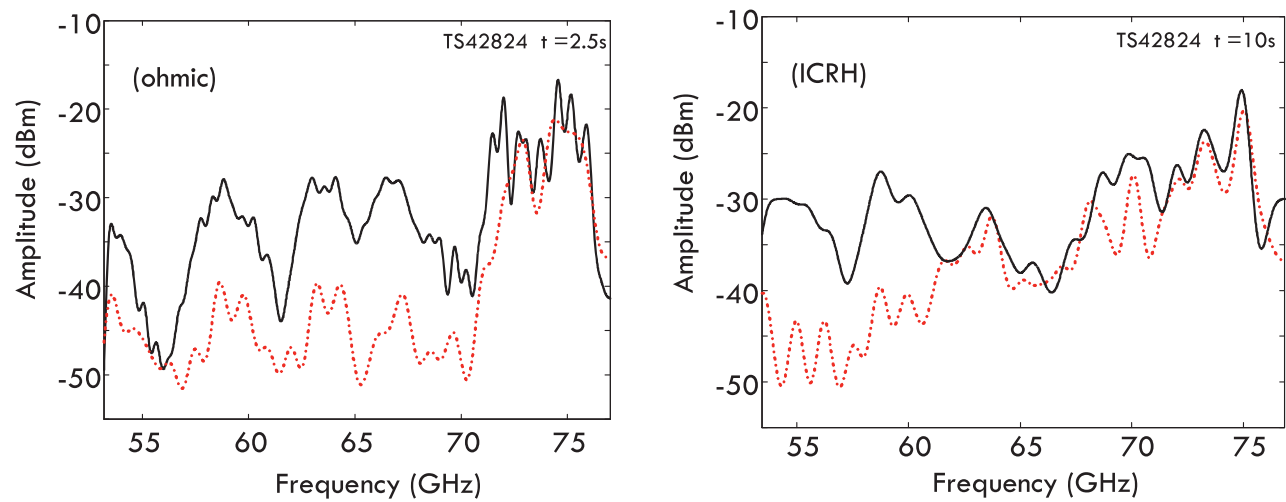

FIG. 5. (Color online) Reflected signal amplitude comparison using tomography analysis (dashed line) and band-pass filter 5-19 MHz (solid line), for ohmic (left), and ICRH (right). 


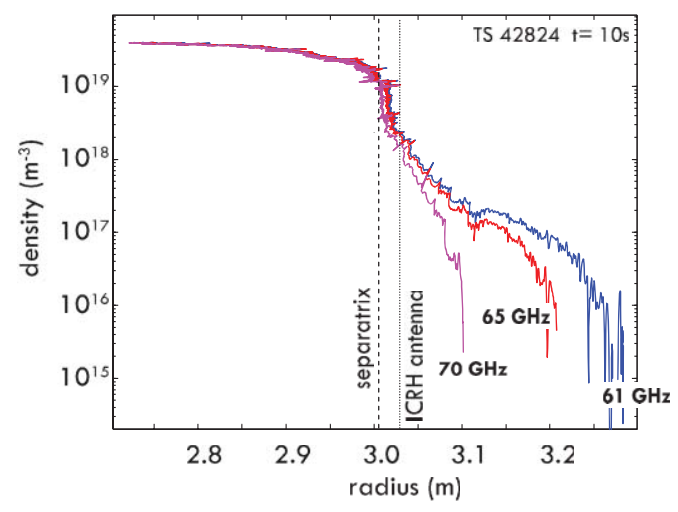

FIG. 6. (Color online) Density profile modifications according the first cutoff frequency used for the profile initialization during ICRH heating phase.

frequency starts to decrease. It is no longer linear and single valued, revealing a strong turbulent zone in the SOL located just before the separatrix region.

The tomograms have been calculated and shown in Fig. 2. As explained in Sec. II, to each $\theta$ corresponds a basis of chirps where each element is distinguished by its initial frequency $f_{0}$. The tomogram exhibits the energy density of the signal, $M$, along each projection. Three main components can be observed. The first component situated in the low $X$ region and associated to the porthole reflection, is well separated from the plasma and wall reflections even in the Fourier decomposition $(\theta=\pi / 2)$. This component is thus readily filtered out in our analysis. In the Fourier space (see Fig. 1) two other components overlap in the vicinity of $20 \mathrm{MHz}$ and are thus impossible to separate. The benefits of the tomogram are shown here as these two parts of the signal form clearly two distinct regions in the range $\theta \in[\pi / 5, \pi / 10]$. We have chosen the particular value $\theta=\pi / 5$ as it can also provide the narrower band pass filter to isolate the plasma signal and improving the signal to noise ratio as well. The projections of the tomograms for $\theta=\pi / 5$ are plotted in Fig. 3, where the different regions of the $X$ axis used for the reconstruction are shown. The three regions correspond, respectively, to the quartz window, the plasma, and the back wall reflected echoes.

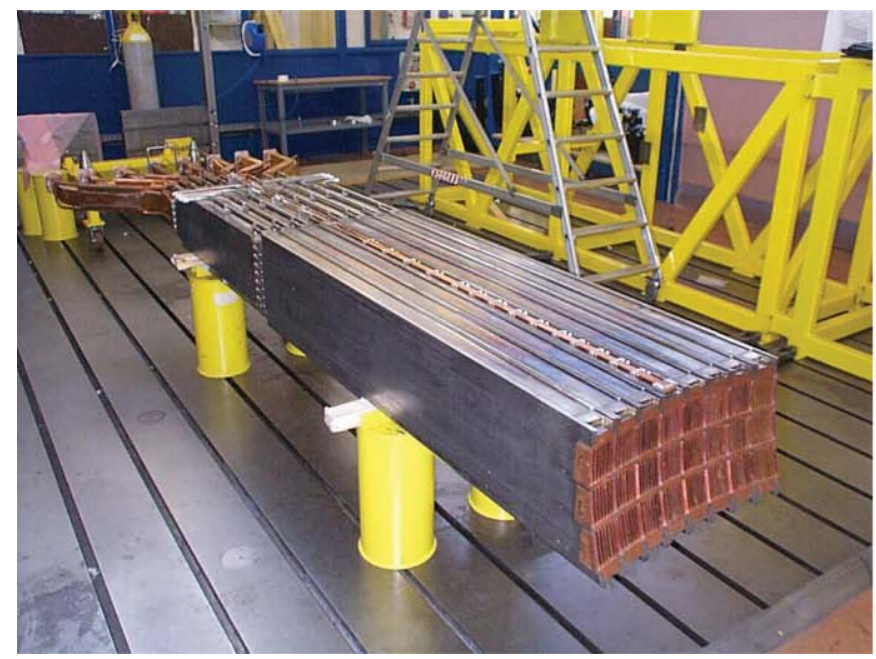

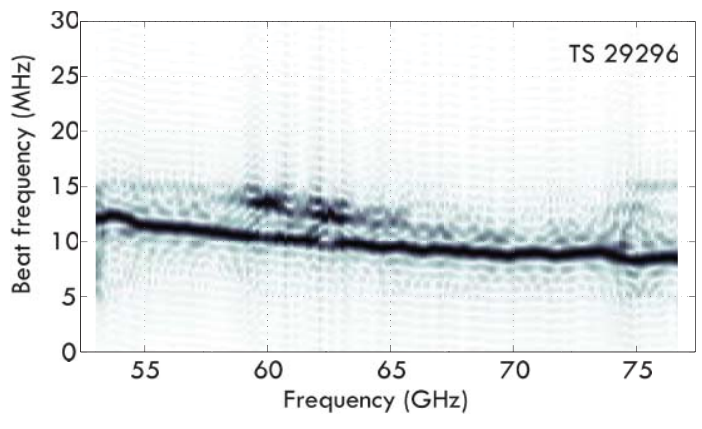

FIG. 8. (Color online) Sliding FFT analysis of the waveguide coupling signal used for reference without plasma.

Thus filtered, the signals can provide a clear plasma echo. Figure 4 shows the FFT spectrogram for comparison with Fig. 1. We can see the effect of the result of the tomographic analysis as it filters the signal with a "timefrequency angle." The phase can now be recovered without ambiguity.

Using only a band-pass filter of 5-19 MHz, in both ohmic and heating conditions, the first cut-off frequency detection from the amplitude rise (Fig. 5) is not straightforward without some additional elaborated filtering. It becomes easier with a tomogram analysis which provides a better sensitivity to the first cut-off frequency determination in lowering the detection limit from -25 to $-35 \mathrm{dBm}$. Thus, it greatly improves the reliability of the density profile initialization.

During ICRH, the first cut-off frequency initialization, while not critical for the core profile reconstruction for frequencies up to $70 \mathrm{GHz}$, has a strong effect on the SOL density profile. Figure 6 shows the density profile reconstruction as a function of the first cut-off frequency in logarithmic scale which exhibits the differences at the far edge plasma. These cut-off frequencies have been determined from different threshold used for the amplitude rise detection. It reveals as well the sensitivity of the diagnostic to densities as low as $10^{16} \mathrm{~m}^{-3}$.

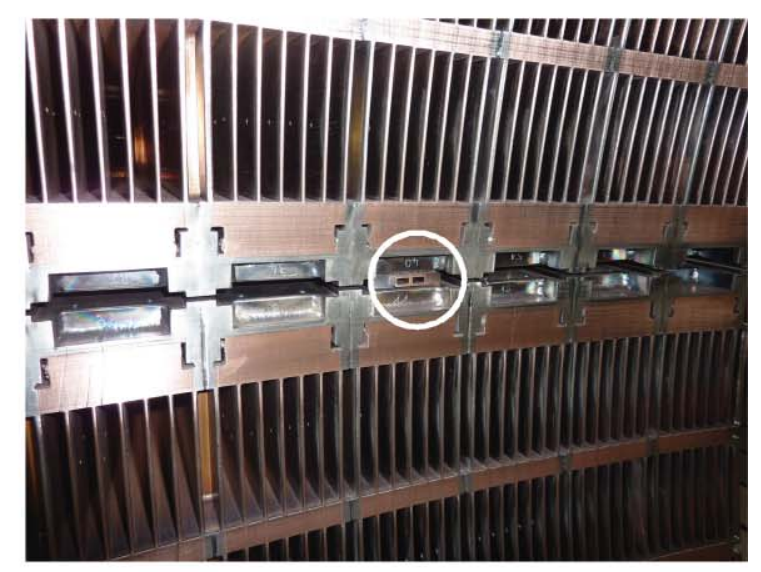

FIG. 7. (Color online) On the left picture is shown the $4 \mathrm{~m}$ reflectometry Ka waveguides located on top of the bottom half of the LH antenna launcher. On the right picture is a closer view of the reflectometer open waveguides facing the plasma in the middle of the full LH coupler. 


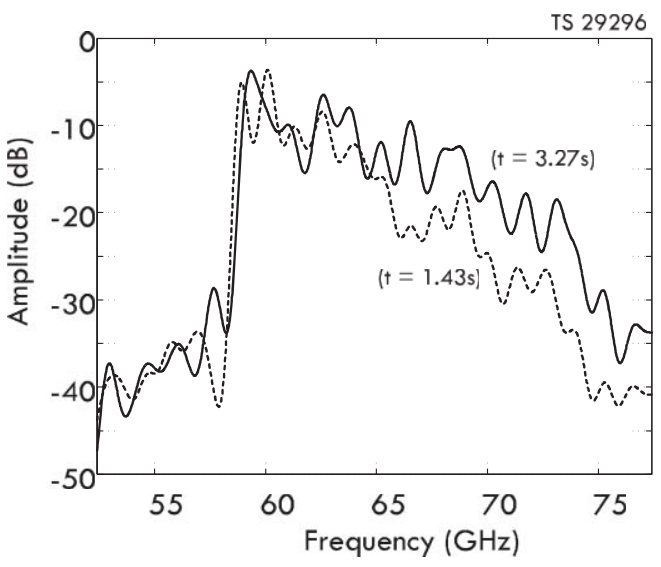

FIG. 9. Amplitude of the reflected signal onto plasma vs. probing frequency. The strong amplitude rise provides a straightforward determination of the first cut-off frequency and corresponds to the calculated electron cyclotron frequency (dashed lines) at the position of the reflectometer waveguide outputs in the LH antenna.

\section{REFLECTOMETRY AND LHCD LAUNCHER ARRANGEMENT}

The edge density profile is a key parameter to address the additional power coupling efficiency. ${ }^{15,16} \mathrm{RF}$ heating methods need special diagnostics for a better understanding of the plasma-wave interaction. Of particular interest is the influence of the wave on the edge plasma in the direct vicinity of the RF heating antenna. One important parameter in this context is the electron density profile, which can be measured by means of microwave reflectometry. First, attempts of density profile measurement by reflectometry were performed, some time ago, near a LHCD antenna and behind the faraday screen of an ICRH antenna ${ }^{17,18}$ to assess profile modification during heating.

Tore Supra uses lower hybrid waves for plasma heating and current drive. ${ }^{19}$ A total amount of power of $10 \mathrm{MW}$ injected by two launchers is actually existing. The coupling of the LH wave to the plasma is a crucial issue, in particular, for the next step device, ITER. ${ }^{20}$ The presence of a cut-off density, $n_{\mathrm{co}}$, below which the LH wave does not propagate, necessitates the ability to control the electron density in front of the LH launcher. The cut-off density corresponds to the density at which the launched wave frequency equals the local electron

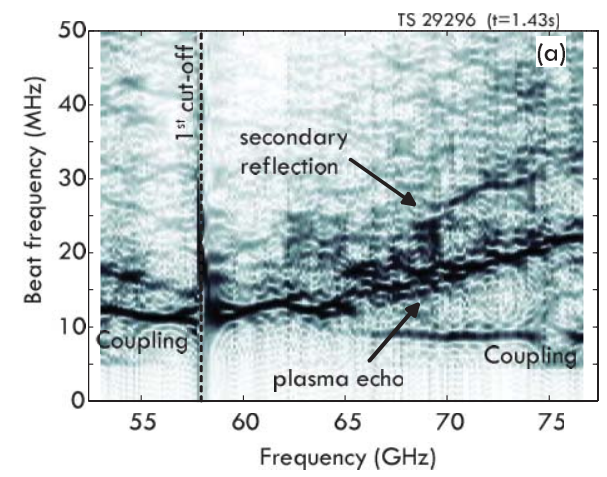

plasma frequency. This gives $n_{\mathrm{co}}=0.0124 \times f^{2}$, where $f$ is the launched wave frequency. For the LHCD system in TS, which operates at $f=3.7 \mathrm{GHz}, n_{\text {co }}$ equals $1.7 \times 10^{17} \mathrm{~m}^{-3}$. In the present day machines, the appropriate electron density for coupling conditions (typically $2-5 \times n_{\mathrm{co}}$ ) can be obtained by moving the launcher or the plasma radially during the pulse. Direct measurements of density in front of the grid are usually conveniently given by fixed Langmuir probes. ${ }^{21}$ This diagnostic offers the advantage of having low cumbersome features; however, it provides a single radial point measurement. Moreover, due to the very low LH cut-off density, measurement in the private zone of the launcher is highly demanded. Results presented in this paper have been obtained with the fast sweep heterodyne V-band $(50-75 \mathrm{GHz})$ reflectometer installed within the coupler for a short period of time. Only few plasma discharges have been realised at low magnetic (3T) to fit the first cut-off frequencies to be in the range of Tore Supra V-band reflectometer. No specific studies on LH coupling physics were undertaken yet.

In order to perform reflectometry measurements in front of the LH antenna launcher, two Ka-band waveguides for emission and reception electromagnetic probing waves have been installed. These waveguides (Fig. 7) are located in the middle of the launcher antenna in the equatorial plane and cover $4 \mathrm{~m}$ through the launcher to the rear where the reflectometer is connected. Quartz window transitions guarantee the vacuum isolation between the reflectometer box and the waveguides.

Due to the low space availability inside the LH antenna, the probing waves are emitted and received directly through open waveguides. Because of the low directivity due to the arrangement of the open waveguides, a coupling signal exists between the waveguides where the emitted probing wave goes directly to the receiving waveguide. Its amplitude is about $35 \mathrm{~dB}$ lower than the plasma signal but can be observed in Fig. 8, thanks to the high sensitivity of the heterodyne detection. This signal can be conveniently used as a phase reference for the density profile reconstruction signal and corrects plasma measurements from phase dispersion occurring in the system (reflectometry setup + waveguides).

During plasma operation the first cut-off is clearly identified in Fig. 9 by a strong and abrupt amplitude rise of the reflected signal. An example is given for two different plasma

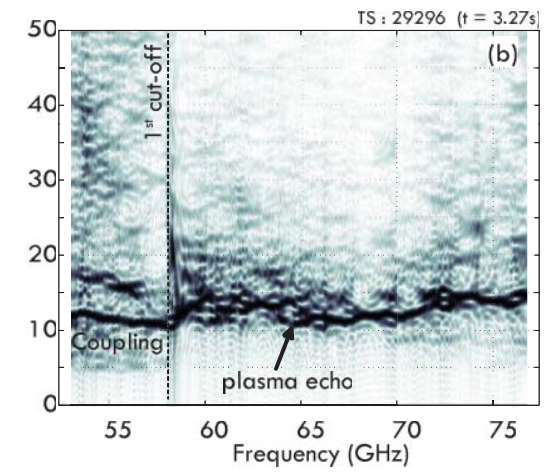

FIG. 10. (Color online) Sliding FFT analysis (amplitude normalized) of the reflectometer data in front of the LH coupler for the two different plasma densities. The vertical dotted line accounts for the first cut-off frequency $\left(\mathrm{n}_{\mathrm{e}}=0\right)$. Main plasma reflection $(X 1)$, secondary echo $(X 2)$, and waveguides coupling can be visualized. Each FFT windows have been normalized to 1 in order to make the phase more visible at the end of the sweep. 


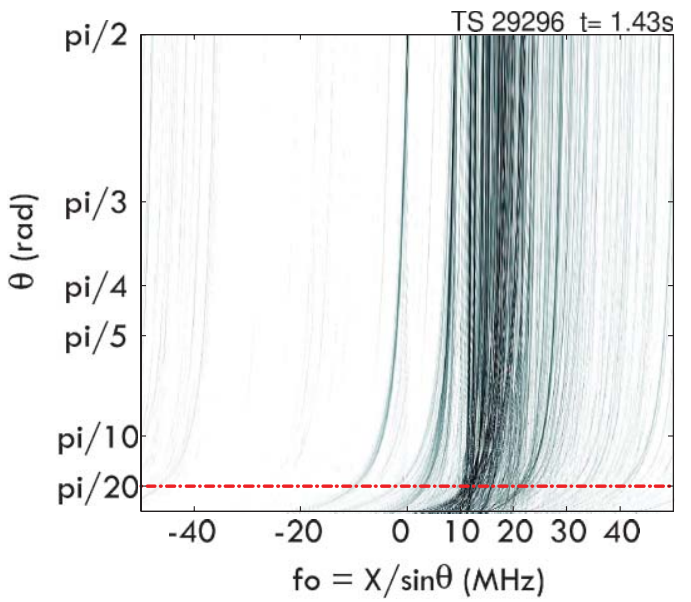

FIG. 11. (Color online) Tomogram of the signal in the LH reflectometer configuration. The horizontal dashed line at $\theta=\pi / 20$ represents the value used for the tomographic decomposition.

density $\mathrm{n}_{\mathrm{e}}(0)=1 \times 10^{19} \mathrm{~m}^{-3}(t=1.43)$ and $\mathrm{n}_{\mathrm{e}}(0)=3$ $\times 10^{19} \mathrm{~m}^{-3}(t=3.27 \mathrm{~s})$. The first theoretical X-mode cutoff frequency $\left(\mathrm{n}_{\mathrm{e}}=0\right)$, which equals the electron cyclotron resonance corresponds remarkably well to the calculated one at the LH antenna radial location. This result provides a good test for the magnetic field calculation required for X-mode reflectometry density profile determination. After the first cut-off, when increasing the probing wave frequency, the amplitude signal decreases substantially because of the low directivity of the emitting/receiving open waveguide system.

The echoes detected on the plasma can exhibit different behaviour (Figs. 10(a) and 10(b)) according the plasma density which modifies the distance between the antenna and the cut-off layer, and modifies as well the influence of the density fluctuations which are less disturbing on the phase signal as the density gradient is getting steeper. ${ }^{22}$ Before the first cut-off, the antenna coupling signal is clearly visible at approximately $-35 \mathrm{~dB}$ below the highest plasma echo. Above $62 \mathrm{GHz}$, multi-reflections can be distinguished in Fig. 10(a) from the main reflection and are dramatically close to each other. Moreover, in this case, the phase fluctuations created by the plasma edge turbulence make it more difficult to distinguish between both echoes. The high density signal case (Fig. 10(b)) provides a rather nice echo and can be treated as in a standard density profile reflectometer.

Applying directly the tomogram analysis is not efficient on this type of signal. First, as seen on the spectrogram of Fig. 10(a), the beat frequency exhibits different behaviours along time and the multi-reflections to be extracted only appear above $62 \mathrm{GHz}$. Second, the amplitude of the signal decreases dramatically (Fig. 9) in the second half of the sweep: time-frequency regions at the end of the sweep are masked in the tomographic projection due to their lack of energy. To remedy these two problems, we have applied the tomogram projection on the truncated signal from $63.5 \mathrm{GHz}$ to the end and we have normalized the amplitude by computing the moving average over 30 points. The tomogram plot is given in Fig. 11. Around $\theta=\pi / 20$ the energy density seems to converge to a small region around $10 \mathrm{MHz}$. This suggests that

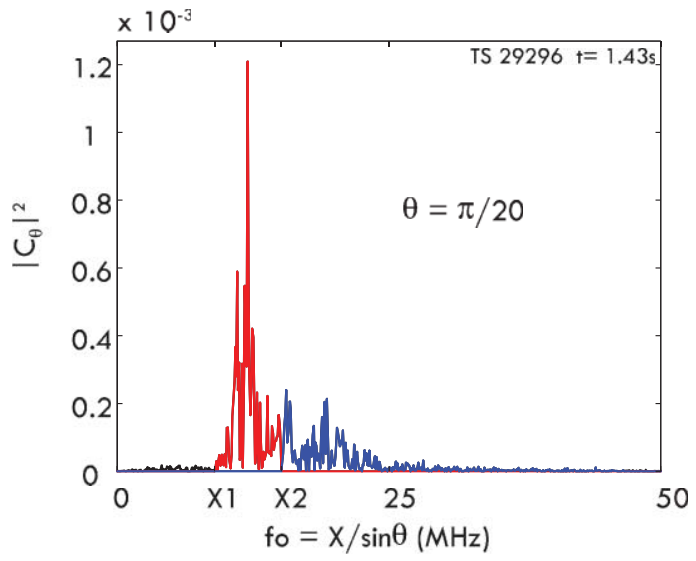

FIG. 12. (Color online) Slice of the tomogram decomposition at $\theta=\pi / 20$ for the LH reflectometer signal. The relevant main plasma reflection is distinguished from secondary echoes.

the slope of the chirps is the closest to the slope of the phase derivative. Thus, $\theta=\pi / 20$ has been chosen for the decomposition. The tomogram slice for this angle is shown in Fig. 12. The signal of interest has its energy located in the highest peak and in a small and fixed neighbouring. The first part (in red) between $X 1<X<X 2$ is then extracted. The region above $X 2$ accounts for the higher order multi-reflections. The region below $X 1$ contains the coupling signal. In Fig. 13 are represented the beat frequencies of the filtered signal and the ones of the signal issued from the whole signal. We can distinguish the components related to the main plasma echo and its secondary reflection. These signals are recovered among the natural fluctuations which are at a high level in the plasma edge, probably enhanced by the LHCD launcher.

Without filtering, the secondary echoes make the phase signal too high compare with the expect phase recovered from tomographic filtering. On Fig. 14 the smoothed density profiles show that the filtered density profile is thus slightly shifted by about $2-4 \mathrm{~cm}$ toward the edge compared to the one calculated from the full signal. This shift is due to the removal

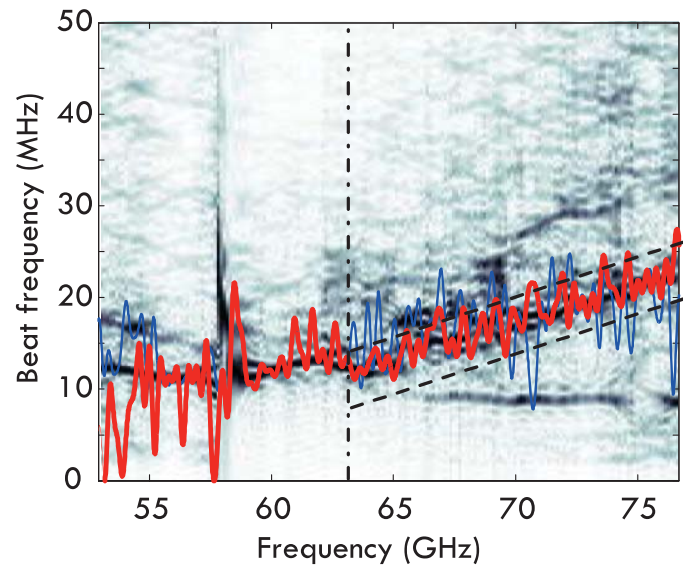

FIG. 13. (Color online) Beat frequency determination of the filtered signal from tomographic analysis (thick solid line) compared to the full plasma reflected signal (thin solid line). The vertical dashed line represents the start of the tomographic analysis. The tilted dashed lines represent the frequency filter bandwidth performed with the tomogram. 


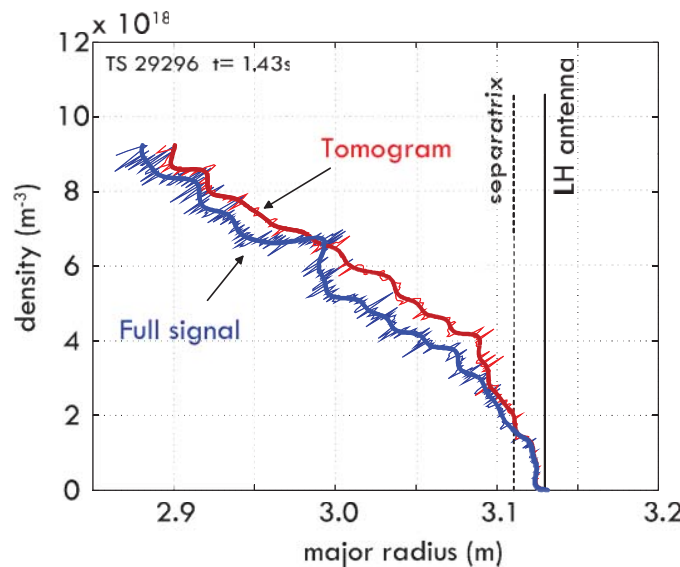

FIG. 14. (Color online) Comparison between reconstructed density profiles with (top profile) and without (bottom profile) tomographic data processing (thick solid lines represent a smooth of the profiles which are provided into the database).

of the secondary echoes (Fig. 13) which make the plasma further away from the LH launcher.

\section{CONCLUSION}

A new data processing tool has been applied to experimental reflectometry signals to provide significant improvements for the extraction of the relevant plasma reflection from the multi-valuated component nature of reflectometry signals. It is now applied routinely into the automatic profile processing from shot to shot for the Tore Supra database. The tomogram provides then an efficient way of filtering out the effect of the walls surrounding the plasma. In the time-frequency plane, by adjusting the tomographic parameter $\theta$, it allows the separation of components having the same frequency range but different time domains. This configuration occurs in reflectometry signals and is discriminated with difficulty using the Fourier transform and band-pass filtering.

For our standard density profile reflectometry while no change on density profile in the confinement region is noticed, it provides a substantial change in the far SOL profile determination. It exhibits a capability for this diagnostic to measure densities down to $10^{16} \mathrm{~m}^{-3}$. Dedicated edge physic ${ }^{23}$ related to heating coupling efficiency or RF sheath processes should benefit of such improvement.

The tomogram decomposition is done using a relatively simple implementation with fast algorithms. It involves basic operations on vectors and the computation of two fast Fourier transforms: one for the projection and one for the reconstruction. This is a huge asset considering the very high number of profiles to be analysed in reflectometry. There is not yet any algorithm able to optimize the choice of $\theta$ for a given signal, in the present study this parameter has been chosen empirically. However, the general shape of the reflections in reflectometry signals is such that the value $\theta=\pi / 5$ seems to be appropriate in the case of a standard density profile reflectometer and the problematic back wall echo is relatively well defined whatever the plasma conditions. The case of the LHCD reflectometer is far more problematic. The tomogram allows better plasma echo isolation from the multi-reflections and substantially improves the density profile reconstruction. We are planning this year to install definitively a new reflectometer in this launcher because the knowledge of the edge density is crucial for the physic of the power coupling. It will be the opportunity to evaluate the tomographic method, which has given us so far the best results, and to test additional tomographic basis. It should provide an interesting experience feedback for the future ITER reflectometer systems expected for the plasma position control and the profile measurement in front of the ICRH antenna.

The presence of a high level of turbulence in the plasma edge creates multi-valuation of the beat frequency. It can greatly complicate the identification of the relevant plasma reflection, whatever the efficiency of the analysis technique. However, the relation between the shape of the turbulence and the type of multi-valuation is an important question to cope with in future research. Up to now, only one point of the timefrequency plane was chosen at each time for the density calculation, leading at best to an averaging of the true density profile and at worse to a false estimation. The Bottollier-Curtet profile reconstruction method assumes a monotonic increase of the density with respect to the plasma depth and hence does not take into account the fluctuations caused by turbulences. This would require further tests based on numerical simulations.

\section{ACKNOWLEDGMENTS}

This work, supported by the Jean Lamour Institute UMR 7198 French National Center for Scientific Research (CNRS)UHP funds and the European Communities under the contract of Association between EURATOM and CEA, was carried out within the framework of the European Fusion Development Agreement. The views and opinions expressed herein do not necessarily reflect those of the European Commission. The authors also wish thank Dr R. Vilela Mendes for fruitful scientific discussions.

${ }^{1}$ H. Bottollier-Curtet and G. Ichtechenko, Rev. Sci. Instrum. 58, 539 (1987).

${ }^{2}$ F. Clairet, M. Paume, and J. M. Chareau, in Proc. 21st EPS Conf. on Controlled Fusion and Plasma Physics, Montpellier, France, 1994 (ECA 1994), Vol. 18B, Part III, p. 1172.

${ }^{3}$ K. W. Kim, E. J. Doyle, T. L. Rhodes, W. A. Peebles, C. L. Rettig, and N. C. Luhmann, Rev. Sci. Instrum. 68, 466 (1997).

${ }^{4}$ P. Varela, M. E. Manso, and A. Silva, Nucl. Fusion 46, S693 (2006).

${ }^{5}$ P. Moreau, F. Clairet, J. M. Chareau, M. Paume, and C. Laviron, Rev. Sci. Instrum. 71, 74 (2000).

${ }^{6}$ F. Briolle, R. Lima, and R. Vilela Mendes, Meas. Sci. Technol. 20, 105502 (2009).

${ }^{7}$ G. Vayakis, C. I. Walker, F. Clairet, R. Sabot, V. Tribaldos, T. Estrada, E. Blanco, J. Sánchez, G. G. Denisov, V. I. Belousov, F. Da Silva, P. Varela, M. E. Manso, L. Cupido, J. Dias, N. Valverde, V. A. Vershkov, D. A. Shelukhin, S. V. Soldatov, A. O. Urazbaev, E. Yu Frolov, and S. Heuraux, Nucl. Fusion 46, S836 (2006).

${ }^{8}$ F. da Silva and S. Heuraux, Rev. Sci. Instrum. 79, 10F104 (2008).

${ }^{9}$ A. Borthwick, G. Agarici, A. Davis, P. Dumortier, F. Durodie, J. Fanthome, C. Hamlyn-Harris, A. D. Hancock, D. Lockley, R. Mitteau, M. Nightingale, R. Sartori, and K. Vulliez, Fusion Eng. Des. 84, 493 (2009).

${ }^{10}$ V. I. Man'ko and R. Vilela Mendes, Phys. Lett. 263, 53 (1999).

${ }^{11}$ M. A. Man'ko, V. I. Man'ko, and R. Vilela Mendes, J. Phys. A 34, 8321 (2001).

${ }^{12}$ F. Briolle, R. Lima, V. I. Man'ko, and R. Vilela Mendes, Meas. Sci. Technol. 20, 105501 (2009). 
${ }^{13}$ F. Clairet, C. Bottereau, J. M. Chareau, and R. Sabot, Rev. Sci. Instrum. 74, 1481 (2003).

${ }^{14}$ R. Sabot, A. Sirinelli, J. M. Chareau, and J. C. Giaccalone, Nucl. Fusion 46, S685 (2006).

${ }^{15}$ F. Clairet, L. Colas, S. Heuraux, and G. Lombard, Plasma Phys. Controlled Fusion 46, 1567 (2004).

${ }^{16}$ A. Ekedahl, K. Rantamäki, M. Goniche, J. Mailloux, V. Petrzilka, B. Alper, Y. Baranov, V. Basiuk, P. Beaumont, G. Corrigan, L. Delpech, K. Erents, G. Granucci, N. Hawkes, J. Hobirk, F. Imbeaux, E. Joffrin, K. Kirov, T. Loarer, D. McDonald, M. F. F. Nave, I. Nunes, J. Ongena, V. Parail, F. Piccolo, E. Rachlew, C. Silva, A. Sirinelli, M. Stamp, K.-D. Zastrow, and JET-EFDA contributors, Plasma Phys. Controlled Fusion 51, 044001 (2009).

${ }^{17}$ F. Leuterer, F. Soldner, M. Brambilla, M. Munich, F. Monaco, M. Zouhar, R. Bartiromo, A. Tuccillo, S. Bernabei, and C. Forest, Plasma Phys. Controlled Fusion 33, 169 (1991).
${ }^{18}$ G. R. Hanson, J. B. Wilgen, T. S. Bigelow, I. Collazo, A. C. Englnad, M. Murakami, D. A. Rasmussen, and J. R. Wilson, Rev. Sci. Instrum. 66, 863 (1995).

${ }^{19} \mathrm{Ph}$. Bibet, A. Ekedahl, Ph. Froissard, F. Kazarian, E. Bertrand, S. Dutheil, and L. Tanaskovic, Fusion Eng. Des. 56-57, 679 (2001).

${ }^{20}$ S. H. Kim, J. F. Artaud, V. Basiuk, A. Bécoulet, V. Dokuka, G. T. Hoang, F. Imbeaux, R. R. Khayrutdinov, J. B. Lister, and V. E. Lukash, Plasma Phys. Controlled Fusion 51, 065020 (2009).

${ }^{21}$ X. Litaudon, G. Berger-by, P. Bibet, J. P. Bizarro, J. J. Capitain, J. Carrasco, M. Goniche, G. T. Hoang, K. Kupfer, R. Magne, D. Moreau, Y. Peysson, J.-M. Rax, G. Rey, D. Rigaud, and G. Tonon, Nucl. Fusion 32, 1883 (1992).

${ }^{22}$ C. Fanack, I. Boucher, F. Clairet, S. Heuraux, G. Leclert, and X. L. Zou, Plasma Phys. Controlled Fusion 38, 1915 (1996).

${ }^{23}$ L. Colas, D. Milanesio, E. Faudot, M. Goniche, and A. Loarte, J. Nucl. Mater. 390-391, 959 (2009). 